\title{
Os recursos humanos de enfermagem da perspectiva da força de trabalho: análise da produção científica
}

\author{
NURSING HUMAN RESOURCES FROM THE PERSPECTIVE OF WORKFORCE: \\ AN ANALYSIS OF THE SCIENTIFIC PRODUCTION \\ LOS RECURSOS HUMANOS EN ENFERMERÍA DESDE LA PERSPECTIVA DE LA FUERZA \\ DE TRABAJO: ANÁLISIS DE LAPRODUCCIÓN CIENTÍFICA
}

\section{Leide Irislayne Macena da Costa e Silva ${ }^{1}$, Marina Peduzzi ${ }^{2}$}

\begin{abstract}
RESUMO
Trata-se de pesquisa sobre recursos humanos em enfermagem, particularmente na perspectiva da força de trabalho, com os objetivos de identificar a produção científica nacional sobre recursos humanos em enfermagem; analisar, particularmente, as publicações sobre força de trabalho de enfermagem; e elaborar uma série histórica sobre a composição e a distribuição dessa força de trabalho no país. A pesquisa bibliográfica foi realizada nas bases de dados LILACS, PeriEnf, DEDALUS, BDENF, PAHO, MS, ADSAUDE, ENSP, FSP, TEXTOC, SIDORH e nos Catálogos do CEPEn, no período de 1958 a 2001. Identificaram-se 469 publicações, 345 (73,6\%) com o descritor recursos humanos de enfermagem, 79 (16,8\%) e 45 $(9,6 \%)$ respectivamente com os unitermos força de trabalho de enfermagem (FTE) e mercado de trabalho de enfermagem. Observase uma concentração da produção na segunda metade dos anos 80 e toda a década de 90 .
\end{abstract}

\section{DESCRITORES}

Recursos humanos de enfermagem. Força de trabalho. Pesquisa em administração de enfermagem.

\begin{abstract}
The focus of this research is on human resources in nursing, particularly from the perspective of workforce. The objectives are to identify the national scientific production on human resources in nursing, analyze particularly those publications about workforce in nursing, and give a historical perspective on the composition of nursing workforce and the distribution of nursing workers throughout the country. In this bibliographic research LILACS, PeriEnf, DEDALUS, BDENF, PAHO, MS, ADSAUDE, ENSP, FSP, TEXTOC and SIDORH's databases were consulted, as well as CEPEn's catalogues, from 1958 to 2001. 469 publications were identified: 345 (73,6\%) utilized human resources in nursing as a descriptor, while $79(16,8 \%)$ and 45 (9,6\%) utilized, respectively, the keywords nursing workforce (FTE) and nursing work market. There is a higher concentration of scientific production in the second half of the 80's and throughout the 90's.
\end{abstract}

\section{KEY WORDS}

Nursing staff.

Labor force.

Nursing administration research.

\section{RESUMEN}

Este estudio es una investigación sobre recursos humanos en enfermería, particularmente en la perspectiva de la fuerza de trabajo, realizado con los objetivos de identificar la producción científica nacional sobre recursos humanos en enfermería; analizar, particularmente, las publicaciones sobre fuerza de trabajo de enfermería; y elaborar una serie histórica sobre la composición y la distribución de esa fuerza de trabajo en el país. La investigación bibliográfica fue realizada en las bases de datos LILACS, PeriEnf, DEDALUS, BDENF, PAHO, MS, ADSAUDE, ENSP, FSP, TEXTOC, SIDORH y en los Catálogos del CEPEn, en el período de 1958 al 2001. Se identificaron 469 publicaciones, 345 (73,6\%) con el descriptor recursos humanos de enfermería, 79 $(16,8 \%)$ y $45(9,6 \%)$ respectivamente con los unitérminos fuerza de trabajo de enfermería (FTE) y mercado de trabajo de enfermería. Se observa una concentración de la producción en la segunda mitad de los años 80 y toda la década del 90.

\section{DESCRIPTORES}

Personal de enfermería.

Fuerza de trabajo.

Investigación en administración de enfermería.
1 Aluna da Escola de Enfermagem da Universidade de São Paulo (EEUSP), bolsista do Programa Institucional de Bolsas de Iniciação Científica (PIBIC/CNPq/ USP-2002/2003). lady.iris@bol.com.br

2 Enfermeira, Professor Doutor da Escola de EEUSP, Departamento de Orientação Profissional, orientadora da pesquisa. marinape@usp.br 
Leide I. M. Costa e Silva Marina Peduzzi

(a) O estudo foi desenvolvido com apoio do Programa Institucional de Bolsas de Iniciação Científica (PIBIC/ CNPq/USP-2002/ 2003) para a bolsista Leide Irislayne Macena da Costa e Silva, e consiste em um sub-projeto da pesquisa intitulada "Avaliação do impacto do PROFAE na qualidade dos serviços de saúde", coordenada pela Prof ${ }^{a}$. Dra. Marina Peduzzi da Escola de Enfermagem da Universidade de São Paulo, Departamento de Orientação Profissional. Essa pesquisa conta com apoio do Ministério da Saúde e da Organização das Nações Unidas para Educação, Ciência e Cultura (Unesco).

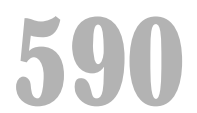

Rev Esc Enferm USP 2005; 39(Esp.):589-96.

\section{INTRODUÇÃO}

Apresentam-se os resultados de um estudo ${ }^{(a)}$ cuja temática central é sobre recursos humanos em enfermagem. Esse tópico é de extrema relevância tanto para a própria área de enfermagem quanto para o campo da saúde, pois dados recentes confirmam que as categorias de pessoal de enfermagem somam $60,2 \%$ dos vínculos de emprego de profissionais de saúde em $2000^{(1)}$. Os autores também assinalam que entre as categorias de pessoal de enfermagem, os auxiliares de enfermagem apresentaram variação positiva de 57,2\% em seus estoques, no período entre 1995 e 2000, ao passo que os atendentes de enfermagem experimentaram variação negativa de $45,8 \%$ e, que no conjunto o pessoal de nível médio de enfermagem experimentou crescimento bruto de 22,1\%.

A referência às categorias de pessoal de enfermagem ao invés de categorias de profissionais de enfermagem, justifica-se pela persistência, embora decrescente, de trabalhadores de enfermagem sem qualificação técnica regular e formal na força de trabalho, do país, embora, como verifica-se acima, os atendentes e similares estejam sendo crescentemente substituídos por auxiliares de enfermagem. Vale ressaltar que esse processo foi ainda mais intensificado desde o ano 2000 com a implantação do PROFAE (Projeto de Profissionalização dos Trabalhadores da Área de Enfermagem) pelo Ministério da Saúde.

Publicação recente sobre monitoramento e avaliação de recursos humanos em saúde da perspectiva internacional, destaca a necessidade e importância da produção de evidências empíricas sobre o tema, como suporte para a tomada de decisão e para a compreensão de tendências ${ }^{(2)}$. Dentre os indicadores referidos pelos autores observa-se a relevância do perfil e de outras características da força de trabalho.

O conhecimento acerca da produção científica sobre recursos humanos também é relevante para o gerenciamento de serviços de saúde e de enfermagem, pois o contingente humano é determinante na produção da atenção à saúde e constitui objeto de intervenção caracterizado pela complexidade. Assim, a gerência de recursos humanos requer a utilização de distintas abordagens e estratégias para ampliar e/ou garantir o alcance dos objetivos dos serviços, o que corrobora com a necessidade de desenvolvimento de estudos tal como este apresentado.

O referencial teórico desta pesquisa está pautado nas linhas de investigação sobre recursos humanos em saúde já sistematizadas na literatura nacional e nos estudos sobre o trabalho de enfermagem.
A pesquisa sobre recursos humanos em saúde conta com importantes contribuições desde os anos 60 e 70, embora seja da década de 80 o início do esforço por institucionalizar a investigação nessa área, particularmente com base no apoio empreendidos pela Organização Pan-Americana da Saúde, Ministério da Saúde, Financiadora de Estudos e Projetos (FINEP) e outras agencias ${ }^{(3)}$.

Desde os anos 60 encontram-se estudos sobre recursos humanos em saúde e em enfermagem, particularmente sobre educação médica e educação em enfermagem $^{(4)}$ o que permite estabelecer a primeira grande linha de pesquisa sobre recursos humanos em saúde, ou seja, formação profissional. Pesquisa desenvolvida por Schraiber e Peduzzi ${ }^{(5)}$ sobre as tendências da investigação de recursos humanos em saúde, verificou que aproximadamente um terço dessa produção teórica encontra-se na categoria formação/capacitação de recursos humanos, o que corrrobora com a temática acima assinalada.

Outras duas importantes abordagens de pesquisa sobre recursos humanos em saúde, aplicável à área de enfermagem, são organização social das práticas / trabalho em saúde e força de trabalho em saúde $e^{(3-5)}$.

No Brasil, os estudos do trabalho de enfermagem foram iniciados em meados dos anos 80 e constituem importante contribuição para a compreensão da enfermagem como prática especializada e, simultaneamente, articulada às demais práticas de saúde ${ }^{(6)}$. Esta abordagem, tal como os estudos do trabalho em saúde, tem contribuído de forma particular na interpretação das questões de recursos humanos de enfermagem tendo em conta os aspectos organizacionais dos serviços de saúde ${ }^{(7)}$.

Na atualidade, encontra-se uma expressiva produção científica sobre o trabalho de enfermagem que será utilizada para a compreensão das características centrais dessa prática e suas tendências, o que, por sua vez, contribuirá para a análise da produção teórica sobre recursos humanos ${ }^{(6)}$.

Quanto a abordagem centrada na força de trabalho, este é um termo consagrado pela economia política, ligado particularmente à escola clássica de Smith, Ricardo e Marx, o qual é, atualmente, utilizado por vários campos científicos no estudo descritivo e analítico de fenômenos demográficos e macroeconômicos ${ }^{(8)}$. O conceito de força de trabalho remete a análise de aspectos gerais e aspectos específicos da divisão do trabalho, da dinâmica de emprego e da qualificação técnica para o trabalho.

Na área de enfermagem encontramos algumas pesquisas desenvolvidas nessa abordagem, que 
representam marcos de referência para o conhecimento do ensino e da prática de enfermagem, no país. O primeiro estudo desse tipo foi realizado pelo Conselho Federal de Enfermagem e pela Associação Brasileira de Enfermagem, no período de 19561958, com a denominação de "Levantamento de recursos e necessidades de enfermagem”(9). Uma segunda investigação dessa envergadura foi realizada no período de 1982-1983, pelos órgãos representativos da enfermagem acima citados, denominado "O exercício da enfermagem nas instituições de saúde do Brasil 1982/1983”, cujo volume 1 foi intitulado “Força de trabalho em enfermagem"(10).

Considerando a relevância das evidências empíricas sobre força de trabalho para as políticas de recursos humanos, o gerenciamento dos serviços e a organização do trabalho, os objetivos deste estudo foram: identificar a produção científica nacional sobre recursos humanos em enfermagem; analisar, particularmente, as publicações da perspectiva da força de trabalho de enfermagem; e elaborar uma série histórica sobre a composição e a distribuição dessa força de trabalho no país.

\section{METODOLOGIA}

Para a consecução dos objetivos propostos foi desenvolvido um estudo bibliográfico ${ }^{(11)}$ sobre a temática recursos humanos em enfermagem, aprofundando particularmente a produção científica identificada na vertente força de trabalho em enfermagem.

Em se tratando de uma pesquisa sobre a força de trabalho em enfermagem no Brasil, o levantamento bibliográfico foi realizado em bases de dados nacionais e latino-americanas. Assim, a busca contemplou as seguintes bases: LILACS, PeriEnf, DEDALUS (global), BDENF, PAHO, MS, ADSAUDE, ENSP, FSP, TEXTOC, SIDORH e os catálogos do CEPEn, disponível em CD-ROM ${ }^{(\mathrm{b})}$.

Os descritores e/ou unitermos utilizados foram: recursos humanos de enfermagem (RHE), força de trabalho de enfermagem (FTE) e mercado de trabalho de enfermagem (MTE). Observa-se uma distinção entre estes termos, particularmente entre descritor e unitermos ou palavras-chave, pois o primeiro consiste numa padronização internacional, tal como ocorre com "recursos humanos de enfermagem” e os demais são utilizados pelos autores para descrever seu objeto de investigação, embora não constituam um padrão de busca bibliográfica de consenso entre as bibliotecas.

A pesquisa abarcou o período de 1958 a 2001, no entanto a busca em cada base de dados variou a depender do período de abrangência da respectiva base. Definiu-se o ano de 1958 para o início do levantamento bibliográfico, pois, como referido anteriormente, nesta data foram publicados os resultados da primeira pesquisa sobre a composição e a distribuição dos trabalhadores de enfermagem ${ }^{(9)}$, que pode ser caracterizada como a primeira investigação da área sobre força de trabalho de enfermagem.

A consulta às bases de dados foi executada no período de dezembro de 2002 e janeiro de 2003.

A análise da produção científica identificada foi realizada em duas etapas. A primeira modalidade de análise foi feita sob a forma de estudo biblio-métri$\mathrm{Co}^{(12)}$, identificando o número de publicações segundo descritor e/ou unitermo, base de dados consultada e distribuição cronológica. Considerando que as publicações podem constar em diferentes bases, foi elaborada uma planilha com autor(es) e título do respectivo trabalho de modo a permitir a eliminação dos repetidos. Assim, procedeu-se a uma sistematização do conjunto do material levantado que permitiu a elaboração de uma panorâmica da produção teórica sobre o tema investigado.

A seguir, os resumos dos trabalhos identificados com o unitermo "força de trabalho" foram analisados visando elaborar uma série histórica com a composição e a distribuição desses trabalhadores. No entanto, não foram encontradas pesquisas que permitissem obter os dados para a série histórica, mas sim estudos que tratam da força de trabalho de enfermagem (FTE) em recortes variados. Estas publicações, identificadas na vertente força de trabalho de enfermagem, foram analisadas por meio da leitura dos respectivos resumos, tendo em vista proceder a uma classificação do material com base nos seguintes aspectos: temática e/ou objeto de estudo, objetivos do estudo e referencial teóricometodológico.

Identificou-se que os resumos das publicações podem não estar disponíveis nas bases de dados, tal como ocorre com o DEDALUS, cuja os resumos de artigos de revista estão disponíveis apenas a partir de 2001. Também observou-se que os resumos podem estar disponíveis parcialmente, ou seja, não estarem publicados na íntegra. Isso ocorreu sobretudo em se tratando de publicações na modalidade de livros, anais de eventos, boletins informativos e teses.

\section{RESULTADOS E DISCUSSÃO}

Inicialmente será apresentada a panorâmica das publicações identificadas na busca bibliográfica,
Os recursos humanos de enfermagem da perspectiva da força de trabalho: análise da produção científica

(b) Bases bibliográficas consultadas: LILACS (Literatura Latino Americana e do Caribe em Ciências da Saúde); Perienf (Base de Dados de Literatura Periódica em Enfermagem); DEDALUS (global) Base de Dados Bibliográficas da USP; BDENF

(Base de Dados em Enfermagem da Biblioteca J. Baeta Vianna da UFMG); PAHO (Acervo da Biblioteca da Organização PanAmericana da Saúde) MS (Acervo da

Biblioteca do

Ministério da Saúde); AdSaude (Administração de Serviços de Saúde); ENSP (Acervo da Biblioteca da Escola nacional da Saúde

Pública); FSP

(Acervo da biblioteca da Faculdade de Saúde Pública da USP); TEXTOC (Textos Completos); SIDORH (Recursos Humanos em Saúde) e CEPEn (Centro de Estudos e Pesquisa em Enfermagem da Associação Brasileira de Enfermagem).

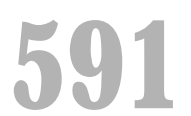

Rev Esc Enferm USP 2005; 39(Esp.):589-96. 
Leide I. M. Costa e Silva Marina Peduzzi segundo descritor e/ou unitermo, base de dados e período de concentração dos estudos.

Na Figura 1 apresenta-se o número de publicações identificadas por descritor e/ou unitermo e observa-se uma diferença entre as publicações com repetição e sem repetição, estas últimas resultando da eliminação dos trabalhos repetidos na mesma base de dados ou entre as distintas bases.

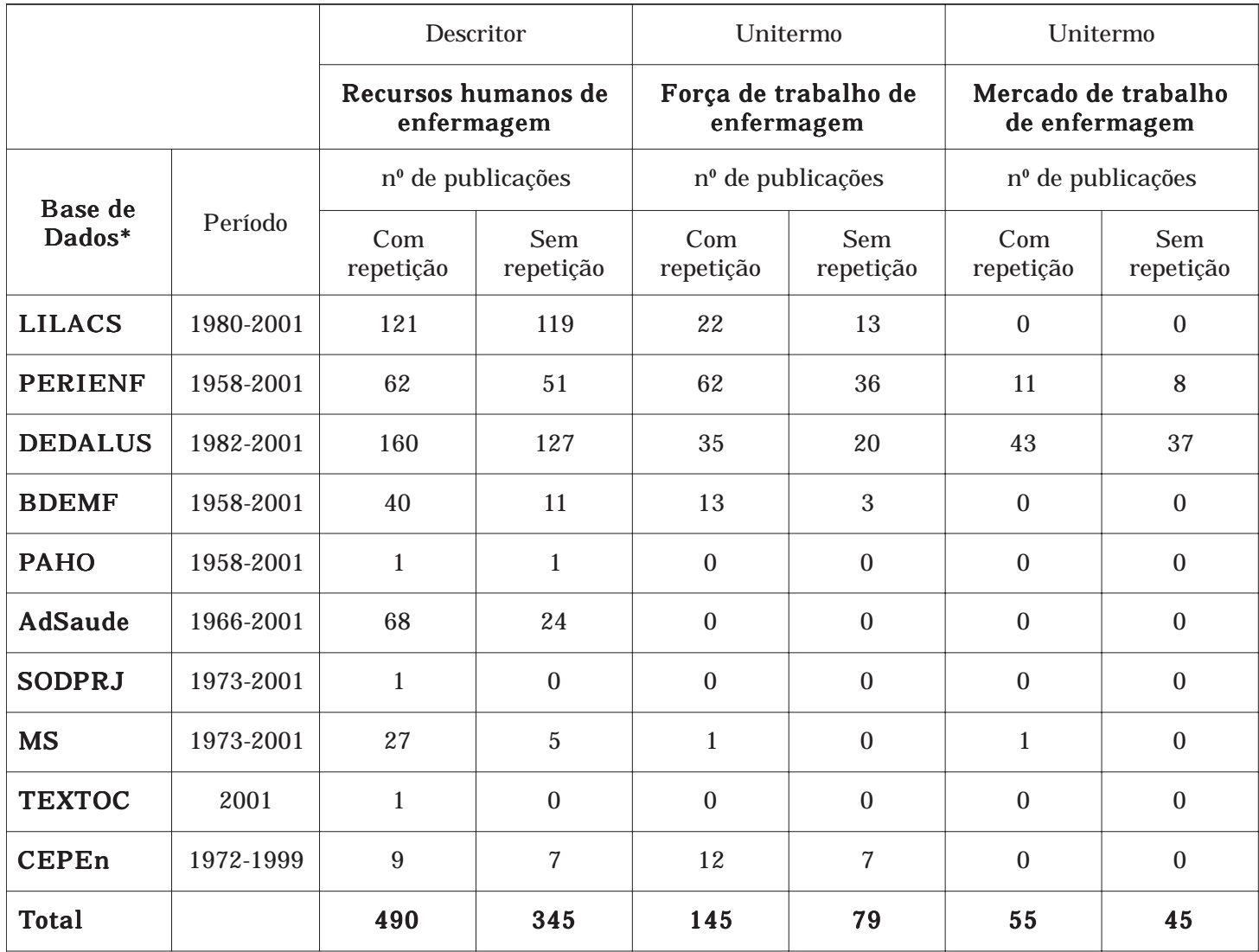

* Na busca bibliográfica realizada nas bases de dados FSP E ENSP não houve resultados, justificando a não inclusão dessas bases na Figura 1.

Figura 1 - Distribuição das publicações identificadas com repetições e sem repetições, segundo base de dados, período e descritor e/ou unitermo. Dez. 2002 a Jan. 2003

A pesquisa identificou um total de 469 publicações sem repetições, no período de 1958 a 2001. Deste total, 345 (73,6\%) foram identificadas com o descritor "recursos humanos de enfermagem", 79 $(16,8 \%)$ com a palavra-chave "força de trabalho de enfermagem” e 45 (9,6\%) com a palavra-chave mercado de trabalho de enfermagem.

Os estudos sobre RHE mostram uma concentração na segunda metade de anos 80 e toda a década de 90. As publicações identificadas com o unitermo FTE mostram a ocorrência de um aumento expressivo a partir de meados da década de 80 que se mantém com uma distribuição uniforme no decorrer dos anos 90, porém com escassa produção quando comparada com aquela referente ao descritor RHE. Foi identificado um número reduzido de estudos sobre mercado de trabalho de enfermagem, com uma significativa concentração apenas no final da década de 90 e anos 2000 e 2001. Portanto, somente nos últimos anos de pesquisa encontra-se uma produção teórica na área de enfermagem sobre o tópico mercado de trabalho.

Conforme previsto, apenas as publicações sobre a vertente força de trabalho de enfermagem (FTE) foram analisadas, tendo sido possível cunhar uma classificação com base na leitura dos resumos. Destaca-se que para a leitura e classificação desse material foi necessário considerar a totalidade de 145 trabalhos identificados com as repetições, conforme consta na Figura 1. Desse total, observou-se que apenas 95 trabalhos apresentavam resumos disponíveis e completos, entre os quais verificouse que havia 17 repetições. Portanto, esse segundo plano de análise foi realizado efetivamente tomando como base 78 resumos, resultando na classificação apresentada na Tabela 1. 
Tabela 1 - Distribuição das publicações sobre força de trabalho de enfermagem segundo categorias de classificação. Dez. 2002 a Jan. 2003.

\begin{tabular}{l|c}
\hline Classificação das publicações sobre FTE & n de publicações $^{\circ}$ \\
\hline FTE e o trabalho/prática de enfermagem & 10 \\
FTE e educação/trabalho de enfermagem & 8 \\
FTE e política de saúde & 8 \\
FTE e mercado de trabalho & 6 \\
FTE e o tabalho/prática do enfermeiro & 5 \\
FTE por Estado & 4 \\
FTE e trabalho feminino & 4 \\
FTE por município & 3 \\
FTE e saúde do trabalhador & 3 \\
FTE e psicodinâmica do trabalho & 2 \\
FTE e emprego em enfermagem & 1 \\
FTE - força de trabalo em saúde & 1 \\
Estudos não pertinentes à categoria FTE & 23 \\
\hline Total & 78 \\
\hline
\end{tabular}

Os recursos humanos de enfermagem da perspectiva da força de trabalho: análise da produção científica
A Tabela 1 mostra que dos 78 resumos, 23 (29,5\%) são estudos não pertinentes à categoria FTE, pois não utilizam o conceito "força de trabalho", tal como consagrado pela economia política clássica, particularmente na teoria do trabalho de vertente marxista. Segundo esta abordagem a força de trabalho consiste no capital variável investido no processo de produção capitalista, à medida que configura-se como fator subjetivo e trabalho vivo em ato que produz um valor suplementar (a mais valia) ao que foi investido (capital constante e capital variável). Assim, a força de trabalho constitui-se em mercadoria ou instrumento do processo de trabalho, que implica no dispêndio de energia; com esforço físico, mecânico e mental do trabalhador ${ }^{(8,13)}$.

A situação identificada e descrita acima, associada à inexistência de descritores exceto "recursos humanos de enfermagem", assinalada anteriormente, corroboram no sentido de mostrar a necessidade de maiores investimentos na sistematização da produção científica de enfermagem nos bancos de dados informatizados, bem como em estudos bibliográficos que possam mapear o estado da arte das diferentes temáticas e as facilidades e dificuldades para a busca.

A maior parte da produção teórica identificada como não pertinente à categoria FTE, refere-se à estudos sobre aspectos gerenciais da enfermagem, portanto publicações que utilizam a categoria recursos humanos tal como conceituada:

Recursos humanos é expressão advinda da ciência da administração e se subordina à ótica de quem exerce algum tipo de função gerencial ou de planejamento, seja no âmbito microinstitucional (órgão público ou empresa privada), seja no macro institucional (por exemplo, planejamento estratégico nacional). Usa-se o conceito de recursos humanos, portanto, em função de propósito explícito de intervir numa dada situação para produzir e aprimorar, ou ainda para administrar esse recurso específico que é a capacidade de trabalho dos indivíduos. Ele é colocado ao lado de outros recursos, como os materiais e financeiros, igualmente suscetíveis de uma utilização "mais racional"(8).

Embora esses estudos utilizam a palavra-chave FTE, observa-se na análise dos respectivos resumos que investigam temas relacionados aos processo de administração e de gerenciamento de pessoal de enfermagem, tais como; dimensionamento de pessoal, seleção e recrutamento, supervisão, avaliação de desempenho e outros.

Para a classificação do restante da produção científica, que efetivamente utilizou o conceito força de trabalho, foi construída uma tipologia, ou seja, categorias para a classificação, a partir dos critérios anteriormente referidos, temática e/ou objeto de estudo; objetivos do estudo e referencial teóricometodológico.

A classificação mostra que o maior número de publicações está concentrado, em ordem decrescente, nas categorias: FTE e o trabalho/prática de enfermagem, FTE e educação/trabalho de enfermagem, FTE e política de saúde, FTE e mercado de trabalho e FTE e trabalho/prática do enfermeiro (Tabela 1).

Assim sendo, observa-se consistência entre o conceito força de trabalho e os aspectos abordados nos estudos - trabalho e prática social, educação, mercado de trabalho e políticas de saúde, pois da perspectiva teórico-conceitual do trabalho todos

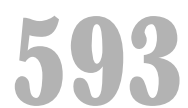

Rev Esc Enferm USP 2005; 39(Esp.):589-96. 
Leide I. M. Costa e Silva Marina Peduzzi esses temas guardam entre si uma relação recíproca, de mútua influência. Pode-se observar uma distinção entre os estudos que tratam da prática de enfermagem e da prática do enfermeiro, os primeiros referidos ao trabalho executado por todos os agentes da enfermagem e os demais, especialmente ao trabalho realizado pelo profissional de nível superior. Embora, ambos digam respeito ao campo de atuação da enfermagem, há diferenças nos processos de trabalho do pessoal de nível médio e dos enfermeiros ${ }^{(14)}$.

Identificou-se escassa produção teórica com o unitermo mercado de trabalho de enfermagem, entretanto, na análise das publicações sobre força de trabalho observa-se a abordagem do mercado de trabalho.

As demais publicações estão distribuídas em categorias que apresentaram reduzido número de estudos, ou seja, quatro ou menos trabalhos por categoria: FTE por Estado, FTE por Município, FTE e trabalho feminino, FTE e saúde do trabalhador, FTE e psicodinâmica do trabalho, FTE e emprego em enfermagem e, finalmente, FTE e força de trabalho em saúde.

Chama atenção que nenhum dos estudos sobre FTE apresente a composição e distribuição dos traba- lhadores de enfermagem no país, mas apenas em relação a alguns Estados da Federação e Municípios.

Considerando a não identificação de produção científica com informações sobre a força de trabalho de enfermagem no país, de modo a permitir a elaboração da série histórica, tal como previsto nos objetivos da pesquisa, foi feita uma consulta ao Conselho Regional de Enfermagem - Seção São Paulo que orientou a busca dessas informações no site do próprio Conselho e/ou no site do Conselho Federal de Enfermagem ${ }^{(15)}$.

Além da busca nesse site também foram obtidos dados atualizados no Anuário Estatístico de Saúde do Brasil ${ }^{(16)}$, que fornece informações sobre força de trabalho de enfermagem com base na Pesquisa de Assistência Médico Sanitária (AMS) da Fundação IBGE (Instituto Brasileiro de Geografia e Estatística), no ano 1999, e informações do Conselho Federal de Enfermagem, no ano 2000.

Essas buscas, somadas às informações que constam na literatura, particularmente a pesquisa desenvolvida pelo COFEN/ABEN em 1982/1983(10) e a pesquisa desenvolvida pelo Ministério da Saúde em $1998^{(17)}$, permitem compor uma série histórica sobre a composição e distribuição da força de trabalho de enfermagem, apresentada na Figura 2.

\begin{tabular}{|c|c|c|c|c|c|c|}
\hline $\begin{array}{l}\text { Fonte e } \\
\text { Período }\end{array}$ & $\begin{array}{c}\text { Censo } \\
\text { COFEN/ABEn }\end{array}$ & $\begin{array}{c}\text { Pesquisa MS/MT } \\
\text { RAIS/CAGED }\end{array}$ & IBGE/AMS & COFEN & $\begin{array}{l}\text { Cadastro } \\
\text { COFEN }\end{array}$ & $\begin{array}{l}\text { Cadastro } \\
\text { COFEN }\end{array}$ \\
\hline $\begin{array}{l}\text { Categoria } \\
\text { Profissional }\end{array}$ & $\begin{array}{c}1983 \\
\text { Brasil }\end{array}$ & $\begin{array}{c}1998 \\
\text { Brasil }\end{array}$ & $\begin{array}{c}1999 \\
\text { Brasil }\end{array}$ & $\begin{array}{l}2000 \\
\text { Brasil }\end{array}$ & $\begin{array}{c}2002 \\
\text { Brasil }\end{array}$ & $\begin{array}{c}2003 \\
\text { Brasil }\end{array}$ \\
\hline Enfermeiros & $\begin{array}{l}25.813 \\
(8,5 \%)\end{array}$ & $\begin{array}{c}70.933 \\
(12,7 \%)\end{array}$ & $\begin{array}{r}70.157 \\
(13 \%)\end{array}$ & $\begin{array}{r}90.020 \\
(14 \%)\end{array}$ & $\begin{array}{l}92.961 \\
(12,7 \%)\end{array}$ & $\begin{array}{l}103.688 \\
(13,4 \%)\end{array}$ \\
\hline $\begin{array}{l}\text { Técnicos de } \\
\text { Enfermagem }\end{array}$ & $\begin{array}{l}19.935 \\
(6,5 \%)\end{array}$ & $\begin{array}{c}70.740 \\
(12,6 \%)\end{array}$ & $\begin{array}{l}49.604 \\
((9,1 \%)\end{array}$ & $\begin{array}{l}102.024 \\
(15,2 \%)\end{array}$ & $\begin{array}{l}111.963 \\
(15,2 \%)\end{array}$ & $\begin{array}{l}144.834 \\
(18,8 \%)\end{array}$ \\
\hline $\begin{array}{l}\text { Auxiliar de } \\
\text { Enfermagem }\end{array}$ & $\begin{array}{l}64.248 \\
(21,2 \%)\end{array}$ & $\begin{array}{l}222.794 \\
(39,7 \%)\end{array}$ & $\begin{array}{l}339.766 \\
(62,8 \%)\end{array}$ & $\begin{array}{l}448.324 \\
(70,1 \%)\end{array}$ & $\begin{array}{l}469.259 \\
(63,8 \%)\end{array}$ & $\begin{array}{c}486.382 \\
(63 \%)\end{array}$ \\
\hline $\begin{array}{l}\text { Atendente de } \\
\text { Enfermagem }\end{array}$ & $\begin{array}{l}194.154 \\
(63,8 \%)\end{array}$ & $\begin{array}{l}115.686 \\
(20,7 \%)\end{array}$ & $\begin{array}{l}82.040^{*} \\
(15,1 \%)\end{array}$ & - & $\begin{array}{l}59.846 \\
(8,2 \%)\end{array}$ & $\begin{array}{l}36.312 \\
(4,7 \%)\end{array}$ \\
\hline Outros & - & $\begin{array}{c}80.500^{* *} \\
(14,3 \%)\end{array}$ & - & - & $233^{* * *}$ & $526^{* * *}$ \\
\hline Total & 304.287 & 560.653 & 541.567 & 640.368 & 734.282 & 771.742 \\
\hline
\end{tabular}

Fontes: Pesquisa COFEN/ABEN 1982/1983; MS, 1999; IBGE/AMS, 2002; COFEN, 2000, 2002, 2003.

Figura 2 - Distribuição da força de trabalho de enfermagem segundo categoria profissional, fonte consultada e período: 1983 à 2003.

A Figura 2 mostra algumas discrepâncias entre as informações fornecidas pelas diferentes fontes, ou seja, a pesquisa MS/MT - RAIS/CAGED (1998) aponta um total de 35\% de atendentes de enferma- gem e similares, enquanto, um ano após, os dados do IBGE/AMS (1999) referem um contingente de $15,1 \%$ de atendentes e os Cadastros COFEN 2002 e 2003 assinalam, respectivamente, um total de ape- 
nas $8,2 \%$ e $4,7 \%$ de trabalhadores de enfermagem sem qualificação técnica formal e regular.

No entanto, publicação recente sobre mercado de trabalho e regulação das profissões de saúde no país ${ }^{(1)}$, apresenta uma análise sobre as dificuldades relacionadas à produção de investigações sobre força de trabalho em saúde, que pode ser estendida à pesquisa sobre força de trabalho de enfermagem e é útil para a interpretação das discrepâncias apontadas. Nesse sentido, os autores assinalam três aspectos: a) escassez de pesquisas e irregularidade na produção de dados estatísticos específicos sobre trabalho e condições de emprego no setor saúde, b) dificuldade de utilização dos dados existentes nos bancos de dados tradicionalmente empregados para análise da FTS e FTE, quais sejam, o inquérito da Assistência Médico-Sanitária (AMS) do IBGE, que é hoje a melhor fonte de dados sobre demanda efetiva de postos de trabalho em estabelecimentos do setor saúde, e a Relação Anual de Informações Sociais e Cadastro Geral de Empregados e Desempregados (RAIS e CAGED) do Ministério do Trabalho e Emprego, que passaram a ser utilizadas a partir de meados da década de 90, c) as diferentes populações investigadas pelas distintas fontes, pois, por um lado, as pesquisas da AMS/IBGE e RAIS/CAGED se limitam ao emprego formal regulamentado, ou seja, abrangem somente os trabalhadores com vínculo empregatício formal, leia-se com carteira de trabalho assinada e, por outro lado, os Conselhos das Áreas Profissionais, tal como o Conselho Federal de Enfermagem [sistema COFEN/COREn(s)], registra todos os profissionais formados através do sistema educacional, independentemente de sua efetiva inserção no mercado de trabalho. Assim sendo, os dados da AMS/IBGE devem estar sub-estimados, pois há um crescimento do trabalho informal também no campo da saúde e enfermagem ${ }^{(1)}$, no país, bem como as informações fornecidas pelo COFEN/COREn-SP podem estar super-estimadas, por contemplar profissionais de enfermagem que não estão efetivamente empregados em estabelecimentos de saúde.

O elevado percentual de atendentes e similares identificados pela pesquisa do MS/MT - RAIS/ CAGED (1999), explica-se pela metodologia empregada que buscava identificar também os trabalhadores de enfermagem sem qualificação técnica regular atuando nos estabelecimentos de saúde, embora com outras denominações de função ${ }^{(17)}$.

As variações observadas na distribuição percentual dos demais agentes da enfermagem (enfermeiros, técnicos de enfermagem e auxiliares de enfermagem) da Figura 2, podem ser atribuídas às diferenças acima apontadas no que se refere aos dados sobre os atendentes de enfermagem e similares. $\mathrm{Na}$ Figura 2, também, pode-se observar que a partir do ano 2000 vem ocorrendo um aumento expressivo de técnicos de enfermagem, bem como um aumento contínuo do contingente total da FTE, no país.

\section{CONSIDERAÇÕES FINAIS}

Apresenta-se uma reflexão sobre três abordagens de investigação da temática recursos humanos - RHE, FTE, MTE, que permite observar a escassez de publicações sobre força de trabalho e mercado de trabalho em enfermagem, com a predominância da abordagem clássica de recursos humanos, tal como concebida na perspectiva administrativa e gerencial. Em parte, isso pode ser atribuído a introdução mais recente dos estudos sobre o trabalho e a tradição mais consolidada de pesquisas sobre o gerenciamento de recursos humanos em enfermagem.

No entanto, a pesquisa mostra que, tal como ocorre no campo de recursos humanos em saúde, na área de enfermagem os autores também vêm desenvolvendo estudos sobre a temática recursos humanos na perspectiva da organização social das práticas/trabalho de enfermagem e da força de trabalho de enfermagem.

A análise das publicações da segunda abordagem, força de trabalho de enfermagem, evidencia que as categorias analíticas trabalho e força de trabalho, vêm sendo utilizadas tanto nas investigações sobre as práticas de enfermagem, quanto especificamente sobre a prática do enfermeiro, o que pode permitir uma maior compreensão das semelhanças, diferenças e contradições entre esses processo de trabalho.

Na busca bibliográfica identificaram-se dificuldades relacionadas à consulta às bases de dados, tais como: a existência de diferenças nos procedimentos de busca em cada base, que requer que o pesquisador se familiarize com os bancos selecionados; a definição de poucos descritores ou unitermos específicos para a produção científica de enfermagem e a ausência ou disponibilidade parcial de resumos dos trabalhos. Nesse sentido, destaca-se a necessidade de maior sistematização e uniformização dos ban cos de dados bibliográficos e da construção de descritores que expressem efetivo consenso entre os pesquisadores, pois isso permitiria maior segurança e abrangência dos dados obtidos.

Considerando que, no total, o maior contingente de publicações refere-se ao descritor "recursos humanos de enfermagem”, será dada continuidade a este estudo através da análise e classificação da produção teórica identificada com esse descritor.
Os recursos humanos de enfermagem da perspectiva da força de trabalho: análise da produção científica 
Leide I. M. Costa e Silva Marina Peduzzi

\section{REFERÊNCIAS}

(1) Girardi SN, Carvalho CL. Mercado de trabalho e regulação das profissões de saúde. In: Negri B, Faria, R, Viana ALD, organizadores. Recursos humanos em saúde: política, desenvolvimento e mercado de trabalho. Campinas: IE Unicamp; 2002. p. 221-56.

(2) Diallo K, Zurn P, Gupta N, Dal Poz M. Monitoring and evaluation of human resourses for health: an international perspective. [on line].Geneva.: Departament of Health Services Provision, World Health Organization; 2003. Available from: $<$ www.human-resources-health.com/content/1/1/3> (30 Apr. 2003)

(3) Nogueira RP. Anotações sobre a trajetória e os problemas de pesquisa em recursos humanos de saúde. Rio de Janeiro: Escola Nacional de Saúde Pública/ Fundação Oswaldo Cruz; 1992.

(4) Ministério da Saúde. Coordenação Geral de Desenvolvimento de Recursos Humanos para o SUS. Seminário de Investigação sobre Recursos Humanos em Saúde. Brasília; 1993.

(5) Schraiber LB, Peduzzi M. Tendências e possibilidades da investigação de recursos humanos em saúde no Brasil. Educ Méd Salud 1993; 27(3):295-313.

(6) Almeida MCP, Mishima SM, Peduzzi M. A pesquisa em enfermagem fundamentada no processo de trabalho: em busca da compreensão e qualificação da prática de enfermagem. In: Anais do $51^{\circ}$ Congresso Brasileiro de Enfermagem; 1999 jul 02-07; Florianópolis. Florianópolis: ABEn-Seção-SC; 2000. p. 259-77.

(7) Nogueira RP. O trabalho em saúde hoje: novas formas de organização. In: Negri B, Faria R, Viana ALD, organizadores. Recursos humanos em saúde: política, desenvolvimento e mercado de trabalho. Campinas: IE Unicamp; 2002. p. 257-73.

(8) Nogueira RP. A força de trabalho em saúde. In: Médici AC, organizador. Textos de apoio - planejamento 1: recursos humanos em saúde. Rio de Janeiro: ENSP/ FIOCRUZ; 1987. p. 13-8.
(9) Associação Brasileira de Enfermagem. Relatório final do levantamento de recursos e necessidades de enfermagem no Brasil 1956 - 1958. Brasília: ABEn; 1980.

(10) Conselho Federal de Enfermagem. Associação Brasileira de Enfermagem. O exercício da enfermagem nas instituições de saúde do Brasil 1982/1983. Rio de Janeiro: COFEN/ABEn; 1985. v. 1.

(11) Gil AC. Como elaborar projetos de pesquisa. $3^{\text {a }}$ ed. São Paulo: Atlas; 1994.

(12) Oliveira JC. Estudo bibliométrico das publicações de custos em enfermagem no período de 1966 à 2000. [dissertação] São Paulo (SP): Escola de Enfermagem da USP; 2001.

(13) Liedke ER. Trabalho. In: Cattani AD, organizador. Trabalho e tecnologia: dicionário crítico. Petrópolis: Vozes; 1997. p. 268-74.

(14) Peduzzi M, Anselmi ML. O processo de trabalho de enfermagem: a cisão entre planejamento e execução do cuidado. Rev Bras Enferm 2002; 55(4):392-8.

(15) Conselho Federal de Enfermagem. Dados estatísticos.[online] Disponível em $<$ www. portalcofen. com.br> (15 set. 2004)

(16) Ministério da Saúde. Anuário estatístico de saúde do Brasil. Brasília: Ministério da Saúde/ Departamento de Informática do SUS/Fundação SEADE; 2002. p. 277-90.

(17) Ministério da Saúde. Secretaria de Políticas de Saúde. Secretaria de Gestão de Investimentos em Saúde. Dossiê: mercado de trabalho em enfermagem no Brasil: PROFAE - Programa de Formação de Trabalhadores na Área de Enfermagem. Brasília; 1999.
Correspondência: Marina Peduzzi Av. Dr. Enéas de $C$ Aguiar, 419 - Cerqueira César - São Paulo 05403-000 - SP

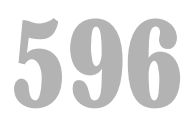

Rev Esc Enferm USP 2005; 39(Esp.):589-96. 